\title{
Une histoire de la coopération agricole de production en
} France

\section{A history of agricultural producer cooperatives in France Una historia de la cooperación agrícola de producción en Francia}

\author{
Serge Cordellier
}

Numéro 331, janvier 2014

URI : https://id.erudit.org/iderudit/1023484ar

DOI : https://doi.org/10.7202/1023484ar

Aller au sommaire du numéro

Éditeur(s)

Association Recma

ISSN

1626-1682 (imprimé)

2261-2599 (numérique)

Découvrir la revue

Citer cet article

Cordellier, S. (2014). Une histoire de la coopération agricole de production en France. Revue internationale de l'économie sociale, (331), 45-58.

https://doi.org/10.7202/1023484ar

\section{Résumé de l'article}

Les coopératives d'utilisation de matériel agricole (Cuma) et les groupements agricoles d'exploitation en commun (Gaec) constituent des formes d'agriculture de groupe relativement bien connues. Il est utile de se pencher sur d'autres formes sociétaires, dont certaines entendaient s'inscrire dans la tradition de la coopération agricole de production (coopération intégrale comparable aux Scop), notamment les " communautés rurales ", les sociétés coopératives agricoles d'exploitation en commun (Scaec) et les coopératives de culture en commun (projet avorté à la fin des années 40). La présente étude passe en revue différentes explications possibles de l'échec ou du très faible impact de ces projets. Ces hypothèses relèvent de registres très différents (politique, social, droit civil, socio-ethnologie...). 


\title{
UNE HISTOIRE DE LA COOPÉRATION AGRICOLE DE PRODUCTION EN FRANCE
}

\author{
par Serge Cordellier*
}

\author{
* Formateur, consultant et \\ chercheur indépendant en \\ économie sociale. Mél.: serge. \\ cordellier@club-internet.fr.
}

Les coopératives d'utilisation de matériel agricole (Cuma) et les groupements agricoles d'exploitation en commun (Gaec) constituent des formes d'agriculture de groupe relativement bien connues. Il est utile de se pencher sur d'autres formes sociétaires, dont certaines entendaient s'inscrire dans la tradition de la coopération agricole de production (coopération intégrale comparable aux Scop), notamment les "communautés rurales", les sociétés coopératives agricoles d'exploitation en commun (Scaec) et les coopératives de culture en commun (projet avorté à la fin des années 40). La présente étude passe en revue différentes explications possibles de l'échec ou du très faible impact de ces projets. Ces hypothèses relèvent de registres très différents (politique, social, droit civil, socio-ethnologie...).

\begin{abstract}
A history of agricultural producer cooperatives in France
CUMAs (Coopératives d'utilisation de matériel agricole, agricultural machinery cooperatives) and GAECs (Groupements agricoles d'exploitation en commun, partnerships of family farms) are relatively well-known types of business groupings in French agriculture. It is therefore interesting to look at some other forms, a few of which are part of the tradition of agricultural producer cooperatives (full-fledged cooperatives comparable to SCOP worker cooperatives), in particular the "rural communities", SCAECs (Sociétés coopératives agricoles d'exploitation en commun, a rare form of farmers' cooperatives) and the "coopératives de culture en commun" (farming collectives proposed by a project that was abandoned in the late 1940s). This study presents various possible explanations for the failure or very limited impact of these projects. These possible explanations draw from a variety of disciplines (political science, sociology, law, socio-ethnology, etc.).
\end{abstract}

\section{Una historia de la cooperación agrícola de producción en Francia}

CUMA (cooperativa de uso de equipos agrícolas) y GAEC (grupos agrícolas para la explotación conjunta) son formas de agricultura en común relativamente bien conocidas. Parece útil de examinar otras formas comunitarias que, para algunos, eran parte de la tradición de la cooperación agrícola de producción, particularmente las "comunidades rurales", las SCAEC (cooperativas agrícolas de explotación en común) y las cooperativas de agricultura en común. El presente estudio analiza las diferentes explicaciones posibles del fracaso o del muy bajo impacto de estos proyectos. Estas hipótesis de explicación pertenecen a registros muy diferentes (político, social, derecho civil, socio-etnología....). 
Les projets de coopération agricole de production ${ }^{(1)}$ ont déçu leurs promoteurs et ont très rarement abouti. Cette affirmation peut étonner. On connaît en effet les coopératives d'utilisation de matériel agricole (Cuma) et les groupements agricoles d'exploitation en commun (Gaec), qui n'ont nullement échoué. Ces deux formes ne relèvent cependant pas, au sens strict, de la coopération de production (coopération de travail intégrale, illustrée par exemple par les Scop, sociétés coopératives ouvrières de production, dans le monde salarié). Nous expliquons ci-après pourquoi et examinons les raisons pour lesquelles les tentatives de coopération intégrale ont déçu leurs promoteurs et eu un très faible impact.

Les Cuma (Chombart de Lauwe, 1952; Rambaud, 1974; Lefèvre, 1996) ont contribué à une modernisation plus solidaire de l'agriculture. Ce sont d'authentiques coopératives, qui, comme en témoignent leurs statuts et leurs pratiques, appartiennent à l'économie sociale et savent se montrer innovantes, mais il s'agit davantage d'une coopération de services entre exploitations (essentiellement pour les machines), sans que le statut de ces exploitations soit modifié.

(1) Cette étude est issue d'une recherche-action engagée par la Fédération nationale des Cuma (FNCuma) sur le thème de la coopération agricole de production, en partenariat avec Coop de France, CER France, Gaec et Sociétés, Trame, l'Inra, la CGScop, la Fédération nationale des centres d'initiative pour valoriser l'agriculture et le milieu rural (FNCivam) et la Fédération nationale de l'agriculture biologique des régions de France (FNAB): www.cuma.fr/documents/ les-racines-historiques-dela-cooperation-agricole-deproduction-pdf. Quant aux Gaec, forme d'agriculture de groupe, ils ont joué et jouent encore un rôle précieux, mais ils ne répondent pas rigoureusement aux principaux critères de la coopération de production. Certes, ils sont notamment caractérisés par l'organisation du travail et la gestion en commun, l'équilibre entre associés et le critère de dimension et de localisation. Chaque associé conserve cependant son statut de chef d'exploitation et les résultats sont partagés en parallèle de la rémunération du travail. Formellement, les Gaec ne correspondent donc pas aux canons de l'économie sociale au sens le plus orthodoxe pour ce qui concerne le caractère non partageable d'au moins une partie des réserves (principe de non-lucrativité); ils représentent davantage la prolongation du statut d'exploitant (ou d'entrepreneur) individuel. L'histoire sociale très riche des Gaec n'en apporte pas moins des enseignements essentiels concernant le sujet qui nous occupe.

\section{Au-delà des Cuma et des Gaec}

L'histoire de la coopération agricole de production en France renvoie à des formes d'organisation autres que les Cuma et les Gaec. Sait-on qu'il existe depuis soixante-cinq ans un statut, la société coopérative agricole d'exploitation en commun (Scaec), de coopération intégrale comme le sont les Scop? Sait-on aussi qu'un projet de " coopératives de culture en commun » a été conçu après-guerre, mais qu'il n'a pas pu être véritablement mis en œuvre? C'est notamment le non-aboutissement de ces formes qui est ici étudié.

Cet article concerne globalement la coopération agricole de production. Il s'inscrit dans la recherche-action engagée par la FNCuma (FNCuma, 2012), laquelle a choisi une approche large de la mise en commun ou de la mutualisation sous diverses formes des moyens de production ou d'une partie de ceux-ci. Le sujet devait cependant être délimité, sur le plan à la fois historique et géographique, car la matière possède une très riche histoire.

S’agissant de la coopération de production, il est possible de remonter aux fruitières (Mélo, 2012), forme d'organisation coopérative multiséculaire 
de transformation du lait en fromage de Franche-Comté, en Savoie et en Suisse alpine, ou aux usages collectifs de biens communs représentés par exemple, dans la période de transhumance, par les pâturages en montagne (alpage, estive, montagne, chaume) ou encore à certaines traditions forestières historiques. Citons aussi, pour mémoire, les formes communautaires agricoles et rurales idéalistes ou regroupées sous la formule « socialisme utopique » et qui ont en général échoué: les colonies sociétaires prônant l'association du capital et du travail créées en France ou dans le Nouveau Monde (Amériques) par des groupes d'origine européenne s'inspirant de théoriciens comme Charles Fourier (17721837). On peut également évoquer les formes d'organisation communautaires propres à une partie des mondes slaves avant la domination du communisme, telles que la zadruga des Slaves du Sud (Bulgarie, ex-Yougoslavie) ou le mir russe, ou bien les coopératives d'esprit libertaire et républicain fondées par des ouvriers agricoles qui s'étaient approprié des domaines en Espagne, notamment en Andalousie, pendant la guerre civile (1936-1939), avant la victoire du putschiste Francisco Franco. Les formes collectives d'exploitation (aux deux sens du terme) instaurées dans les pays communistes (kolkhozes et sovkhozes soviétiques, communes populaires chinoises, coopératives d'Allemagne de l'Est, etc.), malgré leurs noms, n'étaient pas des coopératives, mais des fermes ou des structures d'Etat. De même, nous n'évoquerons pas les réformes agraires menées dans les pays du Sud ou les formes collectives de production ou de vie villageoise israéliennes liées à des pratiques coopératives (kibboutzim, mosha$\operatorname{vim}^{(2)}$...). Enfin, nous ne développerons pas non plus les différentes

(2) Bobot L., 2011, «Le kibboutz fête ses 100 ans: la fin d'un modèle coopératif?", Recma, $n^{\circ} 320$; Galor Z., Sofer M., 2011, "Evolution passée et récente des villages coopératifs en Israël», Recma, $n^{\circ} 321$ (NDLR). formes d'entraide ou de banques de travail en France, même si nombre d'entre elles ont été et sont encore riches d'enseignements.

Nous examinerons dans une première partie l'élan communautaire très fécond issu de la Résistance et de la Libération (projets de communautés, notamment), puis les obstacles nés de la Guerre froide, ouverte en 1947, qui a porté un coup d'arrêt aux ambitions communautaires. Dans une seconde partie, nous examinerons les différentes hypothèses d'interprétations politiques, sociologiques et anthropologiques de l'échec de la coopération de production intégrale en France.

\section{L'élan de la Libération}

La première étape de cette histoire a correspondu aux projets et aux initiatives favorables à la coopération dans la période qui a suivi la Libération: création de statuts nouveaux, encouragements au développement des Cuma notamment.

\section{Le projet communautaire et associationniste de René Colson} René Colson, qui fut secrétaire général de la Jeunesse agricole catholique (JAC) de 1943 à 1948, avait orienté cette association sur les questions de l'avenir professionnel et du métier d'agriculteur. Avec les jacistes, il s'inquiétait du caractère inéluctable du progrès technique et de la nécessité d'y apporter une solution associative, communautaire, pour éviter la prolétarisation et la concentration capitaliste; d'où l'attention portée aux premières expériences, encore tâtonnantes, d’agriculture de groupe. 
Dans la tradition d'enquête bien ancrée de ce mouvement de jeunesse agricole qui devient mouvement de masse dans les années 50 (Cordellier, 2008a), le Secrétariat des communautés et associations rurales, promu par R. Colson, se propose de recenser les expériences, de permettre des contacts directs entre les différentes équipes, de faire connaître les réalisations en cours et de faciliter les relations avec les pouvoirs publics et les organismes officiels, pour l'étude d'un statut juridique adapté. A la suite des journées de travail organisées les 15 et 16 janvier 1949 (Scir, 1949), il affirme que "les associations de travail [permettraient] de trouver une voie vers l'avenir entre plusieurs écueils : d'une part, pour le petit exploitant, l'impossibilitéde s'adapter au progrès actuel, le risque de se trouver écrasédans la concurrence moderne, de se trouver éliminé de la vie paysanne dans laquelle lui et tous ceux qui l'ont précédé ont grandi et vécu; d'autre part, le développement des grosses exploitations, semblables à des usines, ayant d'un côté le patron et de l'autre les ouvriers prolétaires. Cette solution a bien réussi à résoudre des problèmes techniques, mais elle a créé et amplifié un problème social qui reste préoccupant» (Colson, 1950).

En 1951, sous l'impulsion de René Colson, est créée l'Union des ententes et communautés rurales (UECR), jalon pour le développement de l'agriculture de groupe. On a souvent oublié que, si l'entente était une petite association d'agriculteurs à la manière des futurs groupements agricoles d'exploitation en commun ( $\mathrm{Gaec}$ ), la communauté, alors peu répandue, avait quant à elle l'ambition, rarement précisée, de mettre tous les facteurs de production en commun (travail et capitaux, foncier, bâtiments, machines, cheptel), les agriculteurs étant associés et codécisionnaires, comme dans une Scop ou une Scaec, formes de coopération intégrale (lire infra).

$\mathrm{R}$. Colson décède prématurément la même année, à 38 ans. Il revient à l'UECR de définir les principes qui ont présidé à la création des Gaec en 1960-1962, dans le contexte des lois d'orientation de l'époque. Dans ce prolongement, il s'agit, là encore, d'échapper à la fois à la prolétarisation et au mode de développement capitaliste.

\section{UN TÉMOIGNAGE D'ANDRÉ VIAL}

Lors d'un colloque tenu en juin 2010 à Saint-Jean-la-Vêtre (Loire) en mémoire d'André Vial (1927-2003), natif du Forez, ancien dirigeant de la JAC et ancien président de l'Association catholique de la jeunesse française (ACJF), le chercheur Jean Vercherand (2010) commente une série d'articles publiés dans trois numéros (13 février, 13 mars et 17 avril 1949) du journal professionnel Paysans de la Loire sur le thème « Machinisme et structures d'exploitations ». Dans le numéro du 17 avril, A. Vial explique que, sachant que le mouvement en faveur de la mécanisation ne fait que commencer, la coopérative (comprendre la Cuma) offre de gros avantages pour l'achat des machines, selon l'adage "L'union fait la force », mais que cette formule demande «beaucoup de bon esprit » de la part des associés. Il note par ailleurs que la forme de la communauté est peu répandue. Dans la communauté, ce ne sont pas seulement les machines qui sont en commun, mais tous les capitaux (terres, machines, cheptel, bâtiments), selon le schéma d'une coopération intégrale. Chaque propriétaire devient un associé codécisionnaire. 
Lors de la réunion du Syndicat central d'initiatives rurales (Scir) de 1949 évoquée plus haut, autour de René Colson, du Centre national d'études rurales (Cner) ${ }^{(3)}$, ont été étudiées plusieurs réalisations communautaires. Celle de La Motte-du-Caire (anciennes Basses-Alpes, aujourd'hui Alpesde-Haute-Provence) regroupait sept exploitations. Les femmes, désormais, n'étaient plus obligées de travailler dans les champs. Chaque associé s'était spécialisé selon ses aptitudes: "Le travail est mieux prévu, mieux réalisé et beaucoup plus rémunérateur. » Dans le Lot-et-Garonne, trois

(3) Créé à l'initiative des mouvements d'action catholique de la jeunesse agricole (JAC, JACF), du Mouvement familial rural (MFR, action catholique rurale aînée) et l'association Economie et Humanisme, fondée par des dominicains. foyers venant de Normandie se sont installés en communauté sur une ferme abandonnée. "Leur entreprise tourne très bien au point de vue économique et ils sont enchantés du temps libre qui leur reste. » D’autres exemples ont été présentés, provenant de l'Isère, des Vosges, de la Marne, de l'Oise, et aucune de ces communautés " ne pense revenir à la forme d'exploitation première " (Vercherand, 2010, citant André Vial).

\section{Un contexte favorable à une quête d'émancipation}

Au sortir de la guerre, à la Libération, l'espoir d'une société nouvelle marquait nombre d'initiatives et de réformes dans la plupart des secteurs. On a rarement fait le rapprochement entre, d'une part, la démarche engagée par R. Colson, fondée sur l'idée communautaire et associationniste, et, d'autre part, le développement contemporain de communautés de travail dans le monde ouvrier (Meister, 1958). Pourtant, le parallélisme est patent.

La communauté de travail salarié restée la plus célèbre est celle de Boimondau (boîtiers de montres du Dauphiné), fondée dans les années 40 à Valence par Marcel Barbu (1907-1984), un industriel catholique, ancien séminariste. Michel Chaudy (2008) explique qu'il s'agissait d' "entreprendre autrement ». Il précise que la communauté de travail est beaucoup plus qu'un moyen de production: elle rassemble des hommes, des femmes, des familles qui ont en commun un lieu de production, mais doit apporter à ses membres l'éducation, la formation et des conférences, des activités sportives et culturelles, la réalisation de logements sociaux, ainsi que la participation à l'animation locale (syndicale, politique, spirituelle). Ce faisceau d'ambitions rappelle celui du Familistère de Guise, fondé par Jean-Baptiste André Godin (18081893), qui a rassemblé sur un même site, dans l'Aisne, une usine coopérative, une coopérative d'habitat social, une coopérative de consommation, un théâtre et une piscine (Draperi, 2008). Patricia Toucas-Truyen (2005) explique que Boimondau représentait une "propriété sociale et indivise de telle sorte que jamais [elle] ne puisse devenir individuelle [...]. En 1948 est créée l'Entente communautaire. [En 1951, les communautés de travail] sont une cinquantaine et elles emploient un millier de travailleurs. [...] Ces entreprises visent, d'une certaine façon, à l'émancipation ouvrière. " Ultérieurement, elles prendront le statut coopératif et rejoindront la CGScop.

La parenté du projet initial de l'Union des ententes et communautés rurales, portée par R. Colson, et de l'Entente communautaire, portée par M. Barbu, est marquée par la proximité du vocabulaire employé, leurs ambitions émancipatrices communes et la conscience des acteurs agricoles de s'inscrire dans une démarche comparable. Au demeurant, R. Colson et M. Barbu étaient 
en relation avec des animateurs (ou de futurs animateurs) de la revue Economie et Humanisme, fondée par des dominicains, laquelle s’intéressait à ces initiatives. P. Toucas-Truyen (2005) indique que Boimondau avait éveillé l'intérêt d'animateurs de la revue, le père Louis-Joseph Lebret et Henri Desroche.

\section{De rares expériences de coopération agricole intégrale}

La recherche documentaire et les échanges et entretiens conduits dans le cadre de cette étude montrent que rares ont été les expériences de coopération agricole intégrale. M. Rouleau relève (1951) qu'existaient, au début $\mathrm{du} \mathrm{XX}^{\mathrm{e}}$ siècle, deux coopératives ouvrières de production agréées par le ministère du Travail (et non par celui de l'Agriculture): Les Jardiniers de Paris, à Bourg-la-Reine, Scop fondée en 1901, et L'Horticulture ouvrière, à L’Haÿ-les-Roses, fondée en 1903.

Dans un entretien conduit avec Etienne Lurois, agriculteur de SeineMaritime qui porte activement le projet de création d'une coopérative d'activité et d'emploi (CAE) agricole, Monique Dumas, fille du directeur (1940-1970) de l'ex-société coopérative horticole L'Horticulture ouvrière, explique que "la coopérative employait à temps complet une quinzaine d'horticulteurs qui étaient tous sociétaires, tous rémunérés de la même façon, [et qu'en] pleine saison, il pouvait y avoir beaucoup plus de personnel ». Elle précise aussi que la Scop comptait de trente à trente-cinq sociétaires lors des années fastes des Trente Glorieuses ${ }^{(4)}$.

Au titre des raisons qui ont abouti à la cessation d'activité de la Scop en 1972, M. Dumas considère que la fermeture en 1969 des halles de Paris (pavillons Baltard) au profit de l'ouverture du marché d'intérêt national (MIN) de Rungis a restreint les débouchés traditionnels de la coopérative ouvrière. Elle cite aussi le fait que la TVA sur les produits horticoles est passée en 1981 de 5 \% à 19,6\% (in Les Amis du Vieux L'Hä̈, 2010).

Il existera par la suite, au moins pour une partie de leur activité, quelques rares coopératives de production de type Scop ou sociétés coopératives d'intérêt collectif (Scic) pouvant par exemple être associées à des Cuma ou autres montages sociétaires.

Deux statuts s'inscrivent pourtant totalement dans la logique de l'économie coopérative et de l'économie sociale dans sa définition statutaire: il s'agit du statut-type de la Scaec, issu de la loi coopérative de 1947 et mis à jour en 2010, et du statut-type de la coopérative de culture en commun, issu de la même époque.

Quelques termes comptent particulièrement concernant la Scaec: la coopérative réunit au minimum sept membres et au maximum cinquante associés; peuvent être notamment associés toute personne physique ou morale ayant qualité d'agriculteur ou de forestier dans la circonscription de la coopérative, tout groupement agricole d'exploitation en commun de la circonscription, toutes les associations et tous les syndicats d'agriculteurs ayant avec la coopérative un objet commun ou connexe (autres sociétés coopératives agricoles, union de ces sociétés, société d'intérêt collectif agricole [Sica]...). Concernant les sociétés coopératives de culture en commun, projet avorté, des statuts-types avaient été homologués (Journal officiel du 26 février 1947). 


\section{L'ÉCHEC DES SCAEC}

Le statut de la Scaec a cependant été très peu appliqué. Parmi les quelques rares exemples généralement cités, figure celui de l'Union des jeunes viticulteurs récoltants (UJVR) de Die (Drôme), créée en 1961; les huit vignerons associés produisant de la clairette de Die ont tout mis en commun pour une exploitation unique (Cariou, 2012). La rareté des cas de Scaec et, plus généralement, des cas de coopération intégrale (dont le retrait du projet de coopératives de culture en commun; lire infra), ne manque pas d'interpeller. Plusieurs explications peuvent être avancées, de natures très diverses, examinées dans la seconde partie de cette étude.

Comme d'autres interlocuteurs, Yves Arnaud ${ }^{(5)}$, ancien directeur du Centre d'étude et de développement de l'agriculture de groupe (Cedag) ${ }^{(6)}$, considère que, hormis le maraîchage et la viticulture, activités dégageant une marge à l'hectare plus forte que la " polyculture élevage », la formule Scaec était difficilement praticable. Il relève que l'addition des charges sociales liées aux salaires des associés et des charges forfaitaires à l'hectare était un handicap souvent rédhibitoire: "En gros, c'était un doublement. [...] Si, à l'époque, les

(5) Entretien du 7 septembre 2012.

(6) Le Cedag a fonctionné de 1962 à 2003. Lire notamment Cedag, 1978.

(7) Petitpaysanfinistérien, Tanguy Prigent (1909-1970) s'était investidanslesannées 30 dans la création des foyers paysans (futurs foyers ruraux avec leur mission d'animation rurale et d'accès à la culture). Coopérateur précoce, syndicaliste très engagé dans la Confédération nationale paysanne (CNP), député SFIO élu en 1936, il prit une part active à la création de la CGA, qui allait écarter à la Libération la Corporation paysanne créée par le régime de Vichy. Nommé en 1944 ministre de l'Agriculture du gouvernement provisoire, il occupa la fonction jusqu'en octobre 1947. Outre sa volonté d'encourager le développement des Cuma et de susciter des formes de coopération de production, son bilan est notamment marqué par la promulgation du statut du fermage et du métayage, l'une des plus grandes avancées sociales duXXe siècle pour la petite paysannerie.
Scaec ne se sont pas développées, c'est à cause de cette notion de charges sociales et fiscales. " Selon lui, la viabilité de la Scaec produisant de la clairette de Die a tenu au fait qu'il s'agit d'un produit à très haute valeur ajoutée et dégageant des marges significatives qui permettent de supporter ces charges.

Y. Arnaud se souvient que "certains militants de l'agriculture de groupe du Cedag ont voulu monter des Scaec, mais ont dû se résigner à créer de classiques Gaec ». La question de l'accompagnement social et fiscal des statuts sociétaires est souvent revenue au cours des journées d'étude organisées à l'occasion de cette recherche-développement. Le même interlocuteur insiste très fortement sur la question des réserves impartageables. Il souligne notamment qu' "aujourd'hui, lorsqu'une Cuma se dissout, ou bien dans la pratique la "réserve impartageable" est partagée, ou bien, lorsqu'un jeune rentre, il y a quelquefois dans la pratique quand même des droits d'entrée qui peuvent représenter assez bien la notion d'un rachat des réserves impartageables. Ily aurait donc, sans doute, des ajustements d'ordre réglementaire à faire».

\section{LE RETRAIT DU PROJET DE « COOPÉRATIVES DE CULTURE EN COMMUN »}

Jean Vercherand, s'appuyant sur le journal Paysans de la Loire (2010), évoque l'avortement du projet de « coopératives de culture en commun ", initialement envisagé par l'équipe du ministre de l'Agriculture(SFIO), Tanguy Prigent ${ }^{(7)}$, après la Libération. M. Rouleau (1951) indique cependant que trois coopératives de culture en commun avaient été créées entre 1948 et 1950: la coopérative d'exploitation agricole des alluvions de la Loire, en Loire-Inférieure (actuelle Loire-Atlantique), la coopérative agricole de travail en commun L'Aurore de Saint-Tulle, dans les Basses-Alpes (actuelles Alpes-deHaute-Provence) et la coopérative agricole de Bouron, dans l'Yonne. 
G. Wright (1967) évoque deux projets de réforme présentés par Tanguy Prigent en 1944, dont celui "d'encourager des "coopératives de culture", c'est-à-dire les fusions volontaires de plusieurs exploitations familiales voisines en vue d'une gestion collective (quoique la propriété n'en devînt pas collective). Ces deux projets provoquèrent une tempête de protestations contre les "apprentissorciers" qui, disait-on, étaient en train d'ouvrir la route à un système kolkhozien ». J. Vercherand précise, en s'appuyant sur les archives, que «même les Cuma [étaient] soupçonnées de préparer [de manière rampante] une agriculture collectiviste». Il ajoute que les protestations les plus vives sont venues de la Fédération nationale des syndicats d'exploitants agricoles (FNSEA) d'alors, "qui criait à l'étatisme ». La direction de la FNSEA était dominée par d'anciens responsables de la Corporation paysanne du régime de Vichy, que le ministre Tanguy Prigent démantela, pour la plupart d'idéologie agrarienne et en quête de revanche politique contre les forces issues de la Résistance. Ces dirigeants seront démocratiquement écartés à la fin de la décennie 60 et dans les années 70 par une nouvelle génération issue du Centre national des jeunes agriculteurs (CNJA), en majorité venue de la JAC.

\section{Le contexte très défavorable de la Guerre froide}

Une autre hypothèse mériterait d'être approfondie: la forme coopérative Scaec, comme celle des coopératives de culture en commun, est issue de l'importante législation coopérative de 1947 (loi 47-1775) portant statut de la coopération, cadre juridique général dans un contexte politique en cours d'explosion politique, celui de l'alliance tripartite (socialistes, communistes, démocrateschrétiens réformateurs du Mouvement républicain populaire [MRP]), alliance née de la Résistance et alors que la profession était représentée par la Confédération générale de l'agriculture (CGA), dirigée principalement par des socialistes, des radicaux-socialistes et des radicaux laïques, souvent francs-maçons. L’année 1947 marque en effet le début de la Guerre froide. Les années 50 et suivantes seront très différentes dans leurs ambitions. D’autres formes sociétaires verront le jour, notamment les Gaec au début des années 60, lorsque, sous les auspices de la $V^{e}$ République gaulliste, les interlocuteurs professionnels auront changé: il s'agira désormais du CNJA, dont les dirigeants professionnels seront pour la plupart issus de la Jeunesse agricole catholique (lire l'encadré1, page suivante).

\section{Pourquoi un si faible essaimage?}

Les réponses relèvent de plusieurs registres parfaitement compatibles entre eux: la question de la propriété agricole privée et celle des droits de succession et d'héritage.

\section{La question de la propriété agricole privée}

Pour Yves Arnaud ${ }^{(8)}$, "l'un des articles les plus importants du Code civil de 1804 (le Code Napoléon) concerne la propriété individuelle». Selon le Code civil, «le propriétaire peut jouir et disposer de ses biens de la manière la plus absolue pourvu qu'il n'en fasse pas un usage prohibé par la loi ». Cet extrait peut être complété par un autre, issu

(8) Entretien du 7 septembre 2012. 


\section{Encadré 1}

\section{Une nouvelle donne professionnelle agricole}

Jusqu'en 1947, la Grande Alliance, issue de la Seconde Guerre mondiale et réunissant l'Union soviétique, les Etats-Unis, le Royaume-Uni et la France, a conduit certains pays, notamment les trois derniers, à entreprendre d'ambitieuses réformes structurelles instituant ou consolidant l'Etat-providence.

En 1947, la Grande Alliance laisse place à la Guerre froide. En France, il est mis fin au gouvernement tripartite, initialement parrainé par le général de Gaulle et associant notamment socialistes, communistes et chrétiens-démocrates réformateurs. Les ministres communistes sont écartés du gouvernement. Cette période est également marquée par de violents affrontements au sein des organisations professionnelles (Cordellier, 2008b) et, en 1950, par la scission du mouvement coopératif. Ce dernier, historiquement divisé avant-guerre entre les deux courants rivaux des organisations agricoles Rue d'Athènes et Boulevard Saint-Germain, intitulés désignant les sièges de ces deux courants, s'était unifié à la Libération au sein de la Confédération générale de l'agriculture (CGA) dans l'Union centrale des coopératives agricoles (UCCA).

Issue de la Résistance, la CGA est globalement marquée à gauche, comme le Boulevard Saint-Germain. A peine fondée en son sein en 1946, la Fédération nationale des syndicats d'exploitants agricoles (FNSEA), dominée par d'anciens responsables de la Corporation paysanne de Vichy et de la Rue d'Athènes, politiquement conservatrice, s'efforce d'écarter la CGA et de s'imposer comme seule organisation représentative des agriculteurs. Elle y parviendra rapidement, au terme d'un conflit sans concession (Cordellier, Le Guen, 2008).

En 1950, la Confédération générale de la coopération agricole (CGCA), héritière de la Rue d'Athènes, est désormais séparée de la Fédération nationale de la coopération agricole (FNCA), issue de la CGA et proche du Boulevard Saint-Germain. En 1966, une réunification interviendra (Reymond, 1979). Cela donnera naissance à la Confédération française de la coopération agricole (CFCA), qui se rebaptisera ultérieurement Coop de France.

(9) Entretien du 10 septembre 2012. Cf. également Godreau, 1987. de l'article 7 de la Déclaration des droits de l'homme et du citoyen de 1789 : "La propriété étant un droit inviolable et sacré, nul ne peut en être privé. " Y. Arnaud poursuit: "Et aucun des textes juridiques de France, y compris sur l'urbanisme, n'a jamais remis en cause cet article fondamental. Ils ont simplement "égratigné" l'article "propriété privée", avec des droits de préemption, les plans d'occupation des sols, la loi littorale, etc., mais sans toucher au cœur du Code civil sur la propriété. »

La plupart des autres interlocuteurs rencontrés, notamment Paul Favreau, Marcel Godreau et Jacques Longeot ${ }^{(9)}$, mettent en avant, outre cet aspect concernant le respect de la propriété privée, l'attachement historique, en France, à l'exploitation familiale et à l'exploitation à responsabilité personnelle.

On notera que l'arc-en-ciel des sensibilités agricoles défendait cette petite propriété et l'exploitation familiale, y compris au Parti communiste, à rebours de certaines représentations alors en cours qui percevaient les communistes comme collectivistes et adeptes des kolkhozes de type soviétique 
(la collectivisation forcée a commencé en 1929 en Union soviétique). Se souvient-on qu'au tournant des années 30 les deux principaux dirigeants du syndicat à direction communiste de l'époque - la Confédération générale des paysans travailleurs (CGPT) -, Renaud Jean (1887-1961) et Marius Vazeilles (1881-1973), tous deux députés (très en vue) à l'Assemblée nationale, ferraillaient, au-delà de certains louvoiements circonstanciels ou gages donnés au Komintern (III Internationale, communiste et dirigée par Moscou), contre les collectivisations forcées et la nationalisation des terres et pour la défense de l'exploitation familiale privée?

Y. Arnaud manifeste par ailleurs sa préoccupation face à la financiarisation de l'économie en général et de l'agriculture en particulier, notamment concernant les problèmes des charges de reprise des investissements et la transmission. Il compare cette situation à celle des entreprises bretonnes de transporteurs initialement fondées sous forme familiale et de plus en plus contrôlées par des groupes transnationaux avec perte de l'ancrage territorial. Il plaide en faveur de formes de sociétés civiles pérennes, ce qui suppose l'existence de réserves non partageables, non imposables, pour faciliter la transmission, en l'allégeant.

\section{DES DÉBATS RÉCURRENTS}

Ancien président de la FNCuma, Paul Favreau (Vendée) rappelle ${ }^{(10)}$ les difficultés rencontrées dans les années 80 pour concevoir un rôle élargi des Cuma, au-delà de la mise en œuvre des matériels liés au cycle annuel de production. "Tout ce qu'un agriculteur peut faire individuellement, il doit pouvoir le faire en Cuma, mais il n'était pas facile defaire évoluer la législation. De plus, nous rencontrions de nombreuses incompréhensions ou des barrages au sein même du mouvement, lorsque nous parlions de conditionnement, de transformation, de vente des produits, mais aussi de drainage ou d'aménagement foncier ou encore de travail avec les collectivités pour entretenir leur territoire municipal. [...] Les groupes parlementaires ont fait des projets de loi. Les organisations professionnelles agricoles étaient assez réservées dans leur ensemble. [...] Les agriculteurs cumistes du Sud-Ouest poussaient pourfaire avancer ces dossiers. Ceux des autres régions, l'Ouest par exemple, n'étaient pas chauds pour cette évolution; mais la prise de conscience a progressé lors du congrès de 1993 à l'occasion de visites de terrain de différentes réalisations présentées par les Cuma du Cantal. » Retraité depuis dix-sept ans, P. Favreau constate que les "Cuma ont pris une place dans les mutations d'un milieu rural confronté à des problèmes d'aménagement et d'environnement ».

Marcel Godreau, ancien directeur de la FNCuma, qui a fait équipe avec P. Favreau, émet un jugement convergent ${ }^{(11)}$ : "Peu de responsables, même au niveau de la FNCuma, étaient prêts à aborder cette question du "dépassement" des Cuma. Pour beaucoup, celles-ci étaient [seulement] destinées à accompagner l'exploitation familiale. Pour un certain nombre, le niveau Cuma était difficilement "dépassable", même si cela apparaissait comme souhaitable. C'est avec Jacques Longeot [ancien du Cedag, d'Entraid'Ouest et d'Entraid'Oc] que ce débat était le plus ouvert. Peut-être ne sommes-nous pas allés assez loin et n'avons-nous pas assez mis ce débat sur la table. » J. Longeot ${ }^{(12)}$ insiste sur la "nécessité de développer une double compétence, thématique et territoriale» et pour cela de ne pas rester

(11) Entretien du3octobre2012. (12) Entretien du 10 septembre 2012. 
strictement " calés » sur le statut Cuma: "D’autresstructures (Sica, coopératives de proximité et même entreprises privées classiques) peuvent agir de manière pertinente et il convient defonctionner en réseau, de capitaliser les expériences et les pratiques pour pouvoir les transmettre et accompagner les projets [émergents], l'innovation.» Il plaide pour des approches transversales. Son propos est conforté par les exemples que cite Bernard Mondy ${ }^{(13)}$, enseignant-

(13) Entretien du 10 septembre 2012.

(14) Entretien du 10 septembre 2012.

(15) Entretien du 3 octobre 2012 . chercheur, ancien responsable national de la Fédération nationale des centres d'initiative pour valoriser l'agriculture et le milieu rural (FNCivam), et approuvé par Jean Morère ${ }^{(14)}$. J. Longeot regrette au passage qu'il n'existe plus d'outil comme le Cedag, qui offrait des passerelles entre Cuma et Gaec et avec d'autres formes sociétaires.

\section{LA QUESTION DE LA TERRE}

Marcel Godreau, ancien directeur national de la FNCuma, avance plusieurs explications ou hypothèses concernant ce sujet ${ }^{(15)}$. La terre " a toujours fait l'objet d'un lien très fort avec les paysans. Désir de devenir propriétaire de sa terre par sécurité ou soumission à un propriétaire quand on ne pouvait pas faire autrement (noble, industriel, etc.) ». Il souligne que " toute modification en profondeur de l'acte de production a toujours étéfreinée par cette notion de propriété du sol. Soit parce que le propriétaire ne voulait pas en entendre parler. Soit parce que les périodes de baux des agriculteurs d'un même territoire ne correspondaient pas, ce qui était un obstacle à toute velléité de changement. Soit parce que l'agriculteur propriétaire exploitant avait du mal à imaginer l'indépendance de la propriété du sol par rapport à l'acte de production. Il ne faut pas rêver: il y a toujours eu chez les agriculteurs un réflexe très conservateur de "service de la terre" qui passe avant celui de producteur. Il aurait donc sans doute fallu trouver une solution pour déconnecter cette préoccupation de la terre de la question de la production et "sécuriser" les agriculteurs sur ce point. Nationaliser la terre? La municipaliser? Développer autrement les groupements fonciers agricoles?».

\section{LA VOLONTÉ DE RESPONSABILITÉ PERSONNELLE}

"Les agriculteurs se sont toujours considérés comme des entrepreneurs individuels, et toute politique agricole française depuis un siècle a eu pour objectif de renforcer ce statut, à travers cette défense de l'exploitation familiale. Le salariat a toujours été considéré comme une condition sociale au rabais », constate Marcel Godreau, qui poursuit: "La modernité que nous avons apportée à la solidarité professionnelle par l'entraide et les Cuma, pour tout à fait positive qu'elle ait été, a contribué à conforter le statut de l'exploitation familiale en lui permettant de limiter ses investissements, tout en lui assurant une bonne productivité et des conditions de vie meilleures. »

M. Godreau estime que les Gaec ont constitué une solution intéressante, «qui combinait des approches nouvelles et d'autres plus classiques, destinées à sauvegarder lestatut d'exploitant agricole entrepreneur individuel et responsable [...]». Si les Gaec permettent la fusion de plusieurs exploitations, la décision collective, le partage du travail, «ils assurent la continuitéde l'exploitation individuelle par certains autres aspects: ily a rémunération du capital, le nombre d'associés est limité, le Gaec ne touche pas à la question de la propriétéfoncière, etc. » 


\section{Successions et droit d'héritage}

Outre les explications avancées par Marcel Godreau, nous proposons une hypothèse complémentaire. Elle se fonde sur l'interrogation suivante: au-delà des principes égalitaires d'héritage édictés et proclamés par le Code civil en 1804, ne serait-il pas utile de s'interroger aussi sur les singularités des différentes régions françaises concernant les « coutumes » successorales et le droit d'héritage? Cela concerne notamment les traditions et les représentations historiques relatives à la division ou non du patrimoine à chaque génération. Avec des conséquences durables dans les réalités et les mentalités, comme les « types familiaux " pyrénéen (les " cadets de Gascogne ») et breton (les « cousins à la mode de Bretagne») [Augustins, 1985, 1990].

Les ethnologues, les anthropologues et les historiens du droit se sont de longue date passionnés pour ces questions. Les mécanismes de transmission du patrimoine relèvent selon le cas des coutumes ou du droit, mais il faut distinguer les grands principes censés être intangibles et les pratiques, en certains cas beaucoup moins rigoureuses et s'accommodant de modes de contournement et d'arrangements. Pierre Lamaison (1987) explique ainsi que, dans les zones de montagne ou de moyenne montagne de la moitié sud de la France, par exemple dans les Pyrénées, le Massif central ou en Provence, les « systèmes à maison » sont caractéristiques de par leur régime foncier, leur système héréditaire et leurs stratégies matrimoniales. Il en va autrement en général dans la moitié nord du pays.

\section{LE « SYSTĖME À MAISON »}

Les historiens du temps long et les anthropologues parlent ainsi de «maison». C. Lévi-Strauss en précise la définition (Bonte, Izard, 1992): «La maison est d'abord une personne morale, détentrice d'un domaine composéà la fois de biens matériels et immatériels ». Par " matériel », il faut comprendre la possession d'un domaine réel, avec des sites et des territoires, l'« immatériel » comprenant des noms qui sont des propriétés de maison, des légendes. "Ce n'est donc pas la maison qui appartient aux gens, mais les gens qui lui appartiennent et, avec eux, les biens matériels et immatériels qui doivent rester indivis et conservés pour être transmis aux descendants. »

Il ajoute qu'une maison se perpétue par la transmission de son nom, de sa fortune ou de ses titres, à la condition que cette continuité puisse se traduire dans le langage de la parenté (filiation, notamment) ou de l'alliance ou, le plus souvent, des deux ensembles (Bonte, Izard, 1992, p. 435-436). P. Lamaison souligne que les "systèmes à maison " revenaient à un seul héritier par génération dont la succession abouti(ssai)t à la formation des lignées patrimoniales. C. Lévi-Strauss précise que cette succession unique n'a été possible que parce que toutes sortes de mécanismes ont permis l'éloignement des cadets par leurs aînés. Maurice Godelier (2004) utilise des termes comparables pour évoquer la maison comme système de parenté qui « recourt à la fois à des principes de descendance et à des principes d'alliances ».

\section{LES « SYSTĖMES PAYSANS ÉGALITAIRES »}

En Bretagne, en Normandie et plus largement en France septentrionale (et aussi ailleurs), exist(ai)ent en parallèle des "systèmes fondés sur une égalité plus ou moins stricte» entre descendants. P. Lamaison (1987) indique que, 
dans ces systèmes égalitaires, on pratiquait plus fréquemment les « alliances consanguine proches » dans le cercle des cousinages que dans les « systèmes à maison ".

Ces différences fondamentales dans les systèmes de parenté expliquent aussi peut-être des incompréhensions dans le mouvement Cuma entre responsables de régions différentes lorsqu'était évoquée la question de la coopération agricole de production.

\section{Conclusion}

Léchec en France de la coopération agricole de production ne doit pas être globalement attribué à une réticence du milieu agricole vis-à-vis de l'économie sociale, puisque "l'histoire des organisations professionnelles agricoles avait représenté une histoire d'économie sociale, une histoire associationniste, une histoire solidariste » (Cordellier, Le Guen, 2008). Cet échec de la coopération de production (conçue au sens de coopération intégrale) tient à plusieurs facteurs, dont le principal, au terme de l'enquête qui a nourri cette étude, semble avoir été le déclenchement de la Guerre froide en 1947. Cette rupture majeure dans l'histoire politique de la période a mis un terme à nombre d'intentions de réformes (dont certaines déjà engagées) qui supposaient un large accord et il a polarisé les positions et les affrontements entre camps sans tenir compte des projets déjà formulés, des expérimentations en cours et de certaines aspirations.

Au terme de cette étude, il apparaît aussi, à propos par exemple des Scaec, qu'il a pu exister un manque d'articulation entre les règles concernant les charges sociales et celles relatives aux charges fiscales (addition des charges sociales concernant le salariat et des charges forfaitaires à l'hectare) liées à ce statut et, plus largement, à l'absence ou aux carences d'accompagnement social et fiscal des politiques publiques des formes sociétaires. Plusieurs participants au chantier ouvert par la FNCuma ont souligné avec insistance que cela avait constitué jusqu'alors un handicap pour le développement de la coopération à la production. Nous ne pouvons que rejoindre leur constat. Nous avons aussi avancé une hypothèse complémentaire, celle des formes de succession et du droit d'héritage. L'explication par les modèles familiaux (lignage, systèmes de parenté) constitue en effet l'un des fondamentaux de l'ethnologie et de l'anthropologie. Ces différences permettraient sans doute de mieux comprendre les contrastes qui existent d'une région à l'autre en matière de représentations relatives à la coopération agricole de production. 


\section{BIBLIOGRAPHIE}

Anonyme, 2010, "L'horticulture à L’Haÿ », in Les Amis du Vieux L'Haÿ, n 32, L'Haÿ-lesRoses, décembre.

Augustins G., 1985, « Coutumes successorales et droit d'héritage: un exemple comparatif (Pyrénées et Bretagne au XIX ${ }^{e}$ siècle) ", La terre privée et les conduites patrimoniales de la France rurale, p. 147-156.

Augustins G., 1990, "Les transmissions entre générations dans les sociétés paysannes européennes ", Terrain, revue d'ethnologie de l'Europe, cahier n ${ }^{\circ}$ 5, p. 149-166.

Bonte P., Izard M. (dir.), 1992, Dictionnaire de l'ethnologie et de l'anthropologie, PUF, $2^{\mathrm{e}}$ éd.

Cariou Y., 2012, "Une installation à 20000 euros », Entraid', juin, p. 46-47.

Cedag, 1978, "Agriculture de groupe, bilan et perspectives ", Entraide Ouest, $n^{\circ} 31$, p. 33-37.

Chaudy M., 2008, Faire des hommes libres, Boimondau et les communautés de travail à Valence, 1941-1982, Ed. Repas, Valence.

Chombart de Lauwe J., 1952, "Aspects économiques de l'utilisation en commun du matériel agricole ", Bulletin de la Société française d'économie rurale, IV, Sfer, Paris, 1952, p. 125-185.

Colson R.,1950, Motorisation et avenirrural, Centre national d'études rurales (Cner).

Cordellier S., 2008a, JAC-F, MRJC et transformation sociale, histoire de mouvements et mémoires d'acteurs (1945-1985), MRJC.

Cordellier S., 2008b, "Syndicalisme: de l'unité au pluralisme ", Pour, n 196-197, p. 137-150.

Cordellier S., Le Guen R., 2008, « Organisations professionnelles agricoles: histoire et pouvoirs ", in "L'univers des organisations professionnelles agricoles ", Pour, ${ }^{\circ}$ 196-197, Paris, p. 65-79.

Draperi J.-F., 2008, Godin, inventeur de l'économie sociale: mutualiser, coopérer, s'associer, Ed. Repas, Valence.

FNCuma,2012, Coopération agricole de production, Paris.
Godelier M., 2004, Métamorphoses de la parenté, Fayard, Paris.

Godreau M., 1987, « Place des Cuma dans l'agriculture française: situation, évolutions, perspectives des coopératives d'utilisation du matériel agricole », mémoire de diplôme d'études supérieures d'économie sociale, université Paris-I.

Lamaison P., 1987, «"La notion de maison", entretien avec Claude Lévi-Strauss ", Terrain, revue d'ethnologie de l'Europe, $\mathrm{n}^{\circ} 9$, octobre.

Lefèvre D.,1996, A l'ombre des machines: les Cuma, 50 ans de solidarités locales, Editions Entraid'.

Meister A., 1958, Les communautés de travail en France: bilan d'une expérience de propriété et de gestion collectives, Entente communautaire, Paris.

Mélo A., 2012, Fruitières comtoises, de l'association villageoise au système productiflocalisé, FDCL.

Rambaud P. (dir.), 1974, L'étude est consacrée à quatre formes de coopération de travail: Cuma, Ceta, Gaec, groupements de producteurs, Paris.

Reymond P., 1979, Coopération agricole, le combat pour l'unité, Pays d'ici et d'ailleurs, Paris.

Rouleau M., 1951, «Les coopératives de production ", Bulletin de la Sociétéfrançaise d'économie rurale, vol. III-2, Sfer, Paris, p. 104-108. Syndicat central d'initiatives rurales (Scir), 1949, Communautés et associations rurales, Ed. du Scir, Paris.

Toucas-Truyen P., 2005, Les coopérateurs: deux siècles de pratiques coopératives, Ed. de l'Atelier, Paris.

Vercherand J., 2010, "Le machinisme fait-il évoluer les structures de nos exploitations? ", colloque "André Vial, l'homme et l'œuvre », 11-13 juin 2010, Saint-Jean-la-Vêtre (Loire), in Ô Forez! Bulletin des amis de SaintBônet-le-Château, p. 22-23.

Wright G., 1967, La révolution rurale en France, Ed. de l'Epi, Paris. 